\title{
Bouveret's Syndrome: An Unusual Cause of Gastric Outlet Obstruction
}

\author{
Mahim Koshariya ${ }^{1}$, Sandesh Sharma ${ }^{2}$, Udipt Shringi ${ }^{1}$, Romil Jain ${ }^{1}$
}

Corresponding author:

Dr. Mahim Koshariya, MS,FMAS,FIASGO

Department of Surgery,

Gandhi Medical College and Associated

hamidia hospital Bhopal, India. 462001

E-mail :mahimk2000@yahoo.co.uk

\author{
'Department of Surgery,Gandhi Medical College and Associated hamidia hospital Bhopal, \\ India \\ 2Department of G.I Surgery, Gastrocare Hospital Bhopal, India
}

\section{ABSTRACT:}

Bouveret's syndrome is defined as gastric outlet obstruction caused by duodenal impaction of a large gallstone which passes into the duodenal bulb through a cholecystogastric or cholecystoduodenal fistula. It is a rare condition that causes significant morbidity and mortality and often occurs in the elderly with significant comorbidities . Individual diagnostic and treatment strategies are required for optimal management and outcome. We describe a case of Bouveret's Syndrome in an elderly patient who was managed successfully through surgery.

Key word: Bouveret's syndrome ,gastric outlet obstruction ,cholecystectomy, gastrotomy

\section{INTRODUCTION}

Bouveret's Syndrome was first described in 1770 by Beaussier. In 1896 the French physician Leon Bouveret (1) published two cases of gastric outlet obstruction due to gallstone impaction in the duodenal bulb. Gallstone ileus accounts for $1 \%$ to $4 \%$ of cases of mechanical bowel obstruction, but may be responsible for up to $25 \%$ of cases in older age groups. The most common location of a calculus causing obstruction is the terminal ileum. More proximal impaction is unusual whereas obstruction of the duodenum accounts for $1 \%-3 \%$ of all cases. We describe a case of Bouveret's Syndrome in an elderly patient with comorbidities presented with abdominal pain and vomiting of recent onset with no remarkable past history of cholelithiasis and with no marked general or systemic examination findings. No distinct feature in routine blood investigations. Diagnosis was made by ultrasound and CT scan abdomen, and confirmed by upper GI endoscopy. Open cholecystectomy and fistula repair with gastrotomy with retrieval of stone was done and patient was discharged without any complication.

\section{Significance / Uniqueness of the case}

Until 2008, up to 300 cases had been reported in the world literature. Morbidity and mortality rates have decreased in recent years but still remain 
high, estimated at $60 \%$ and $12 \%-30 \%$ respectively, due to the advanced age and the comorbid factors of the patients. Being commoner in females (2) with a previous history suggestive of cholelithiasis, our case of male patient without any remarkable past history suggestive of cholelithiasis and the initial presentation directly in the form of Bouveret's syndrome makes this report of special concern.

\section{CASE REPORT}

A 70 year old male presented with complaint of upper abdominal pain and vomiting for 5 - 7 days duration. He was a known case of diabetes mellitus, hypertension, ischemic heart disease and was under treatment. Per abdominal examination revealed mild epigastric tenderness without any abdominal distension, guarding or rigidity. No palpable mass or organo-megaly was detected. Bowel sounds were normal in intensity and frequency. Per rectal examination was normal.

Blood investigations were done which were suggestive of mild normocytic anemia and neutrophil leuco-cytosis, minor elevation of creatinine and urea. Liver enzymes (AST, ALT, ALP) were within normal limits. Ultrasound abdomen depicted fluid-filled distended stomach, features of chronic cholecystitis, and an acoustic shadow in the area of first part of the duodenum. CT scan abdomen (fig. 1) - revealed gastric outlet obstruction and a low-attenuating filling defect surrounded by a rim of high-attenuating contrast material in the duodenum, just distal to duodenal bulb.

Upper GI endoscopy (fig. 2) - revealed a impacted stone present in the first part of duodenum. Patient was taken for exploration, Gall bladder was found to be fibrosed and cholecystectomy was done. Duodenal fistula was repaired and gastrotomy was performed to retrieve large gallstone of size of size $7 \mathrm{~cm} \times 4 \mathrm{~cm} \times 4 \mathrm{~cm}$ (fig. 3 and 4). No complications occurred after surgery and the patient was discharged on the $8^{\text {th }}$ postoperative day. Biopsy was suggestive of chronic cholecystitis.

\section{DISCUSSION}

Cholelithiasis is a relatively common health problem; by age 75 , about $35 \%$ of women and $20 \%$ of men have developed gallstones (3). Cholecystoenteric fistula complicates $0.3-0.5 \%$ of patients with cholelithiasis. The most common fistula is cholecystoduodenal (60\%) (4), then cholecystocolic (17\%), cholecystogastric (5\%) and choledochoduodenal (5\%). However, more than $80 \%$ of these stones are so small that they are passed in the stools without causing obstruction; only $6 \%$ of the cases

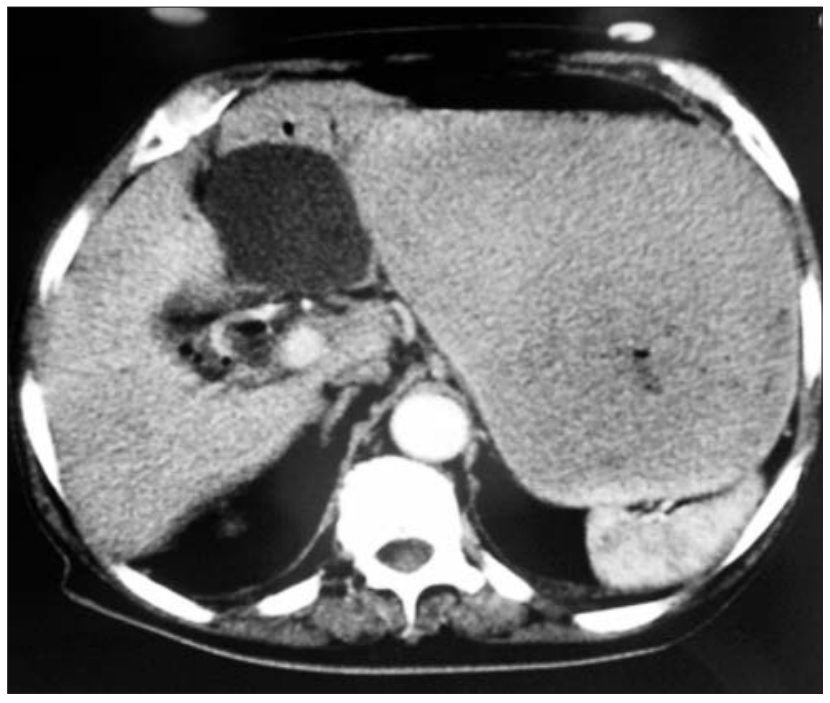

Figure 1 - (Rigler's Triad) gallstone in the duodenum and a distended stomach and pnuemobilia was seen on CT scan

develop clinical intestinal obstruction, defined as gallstone ileus (5) In descending order of frequency, the gallstone can lodge in the terminal ileum (60\%), proximal ileum (24\%), distal jejunum (9\%), and large bowel $(2 \%-4 \%)$. In $1 \%$ to $3 \%$ of cases, the stone obstructs the pylorus and duodenum, causing gastric outlet obstruction, which is known as Bouveret's syndrome. It has a mortality rate of $4.5 \%$ to $25 \%$. The major risk factors for developing this syndrome include age greater than 70 years, female gender, gallstones larger than $2.5 \mathrm{~cm}$ and postsurgical altered gastrointestinal anatomy. In most cases, the presenting signs and symptoms of Bouveret's syndrome are nonspecific. Most common symptoms of patients with Bouveret's syndrome are nausea and vomiting (86\%), and abdominal pain (71\%); less commonly, patients present with hematemesis, weight loss, and anorexia (2) On

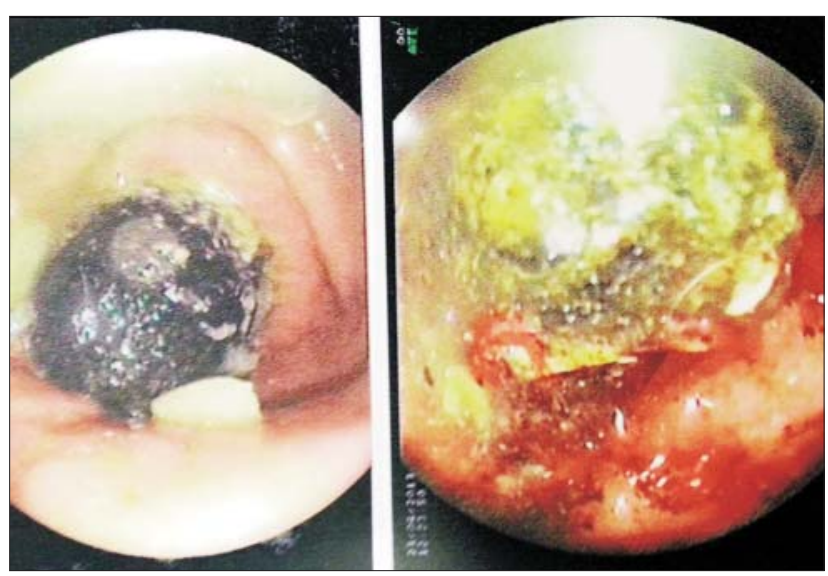

Figure 2 - Endoscopic image showing the impacted gallstone causing obstruction 


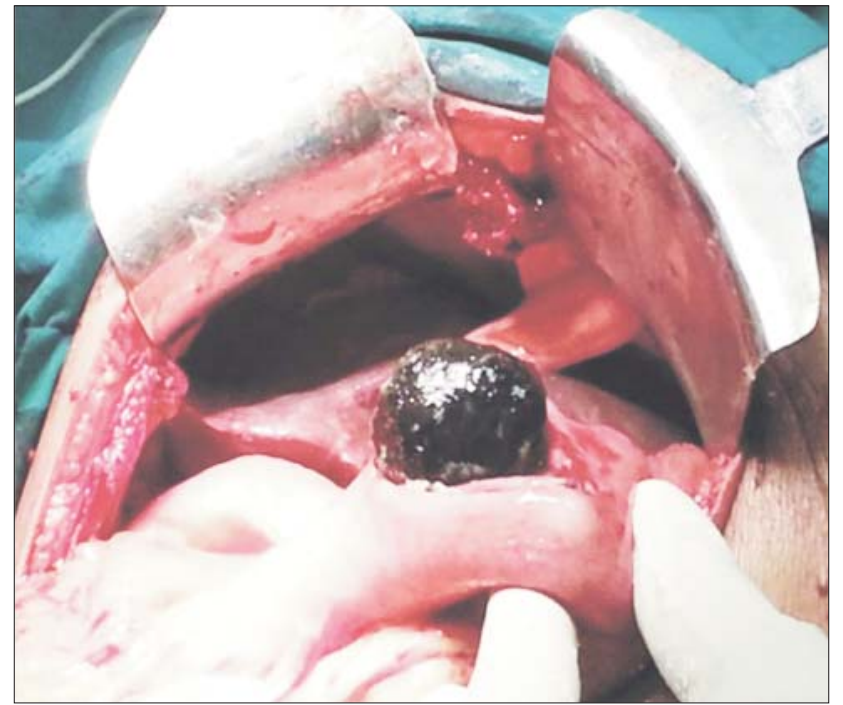

Figure 3- Showing extraction of stone via gastrotomy

physical examination, patients often have abdominal tenderness, abdominal distension, and dehydration. Radiography identifies the classical Rigler's triad of bowel obstruction, pneumobilia, and ectopic gallstones - specific to gallstone ileus - in only $30 \%$ to $35 \%$ of patients whose conditions are later diagnosed as Bouveret's syndrome (6). CT scanning with its superior sensitivity and specificity $(7,8)$, likely represents the best imaging technique to assist in diagnosing Bouveret's syndrome. A potential drawback of CT scanning is that $15 \%$ to $25 \%$ of gallstones appear iso attenuating, relative to bile or fluid. Magnetic resonance cholangiopancreatography (MRCP) may be useful in such cases, because it clearly delineates fluid from calculi. MRCP can also detect the cholecystoduodenal fistula when sufficient fluid is present. Ultrasonographic diagnosis is possible but generally difficult because the gallbladder is usually collapsed, air filled, or both. Without visualization of the gallbladder, the large impacted duodenal calculus could be confused with an orthotropic gallstone showing a wall echo shadow complex, unless the stone is outlined by sufficient fluid in the antro-duodenal lumen.

Gastroduodenoscopy (9) can visualize stones impacted in the duodenum. Extraction of a gallstone via an endoscope $(11,12,13)$ may be a hazardous procedure in patients with large stones because lacerations and perforations may occur. Endoscopic management can not correct cholecystoduodenal fistula and can not recognize any retained stones in the bowel distally. Surgery remains the treatment of choice for gallstone obstruction.

The mortality rates have been quoted to be as high

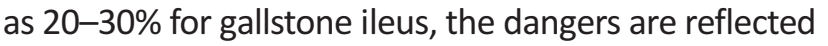

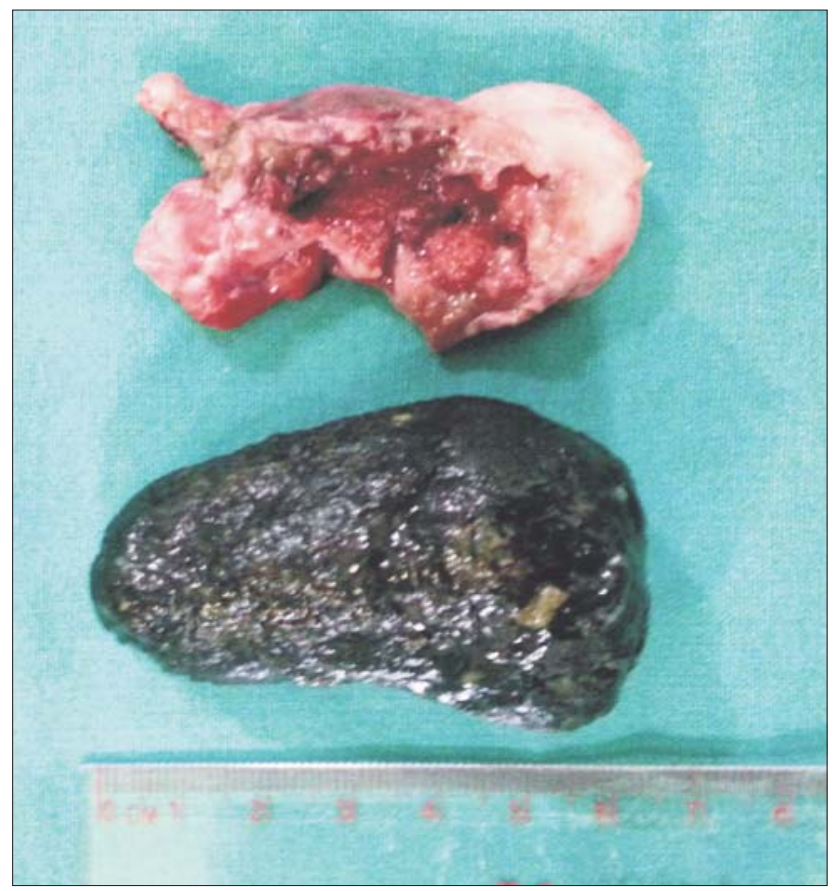

Figure 4 - Fibrosed gallbladder specimen and gall bladder stone of $7 \times 4 \times 4 \mathrm{~cm}$

by the advanced age of the patient and the associate comorbidities. However, controversy exists between simple enterolithotomy or enterolithotomy with cholecystectomy and repair of the internal fistula as a single or one stage procedure. $(14,15,16)$

The potential complications for non-closure of the fistula include cholangitis, cholecystitis, recurrent ileus, erosion of mucosal blood vessels during passage of the gallstone and gall-bladder carcinoma. (17) In our case, we opted for single stage surgery to perform open cholecystectomy, closure of the internal fistula, gastrotomy and removal of $7 \mathrm{~cm}$ stone thus giving a definitive cure and avoiding the future complications of residual fistula.

\section{CONCLUSION}

Though diagnostic dilemmas are there for confirming the diagnosis of bouveret's syndrome especially in elderly patients without any previous history of cholelithiasis or other associated co-morbidities, but once confirmed one should opt for the optimum treatment. We can conclude with our experience in successful treatment of our case that single stage surgery can be considered as an optimum procedure in the treatment of Bouveret's syndrome, provided other associated co-morbidities are under control.

Case was presented at $24^{\text {th }}$ World Congress of IASGO in December 2014 Austria, Vienna. 


\section{REFERENCES}

1. Bouveret L. Stenose du pylore adherent a la vesicule. Revue Medicale (Paris) 1896; 16:1-16.

2. Cappell MS, Davis M. Characterization of Bouveret's syndrome: a comprehensive review of 128 cases. The American Journal of Gastroenterology. 2006;101(9):2139-2146.

3. Newman HF, Northup JD. The autopsy incidence of gallstones. Surgery, Gynecology \& Obstetrics.1959;109(1):1-13.

4. LeBlanc KA, Barr LH, Rush BM. Spontaneous biliary enteric fistulas. Southern Medical Journal.1983;76(10):1249-1252.

5. Pickhardt PJ, Friedland JA, Hruza DS, Fisher AJ. CT, MR cholangiopancreatography, and endoscopy findings in Bouveret's syndrome. American Journal of Roentgenology. 2003;180(4):1033-1035.

6. Frattaroli FM, Reggio D, Guadalaxara A, Illomei G, Lomanto D, Pappalardo G. Bouveret's syndrome: case report and review of the literature. Hepato-Gastroenterology. 1997;44(16):1019-1022.s

7. Tüney D, Çimsit C. Bouveret's syndrome: CT findings. European Radiology. 2000;10(11):1711-1712.

8. Trubek S, Bhama JK, Lamki N. Radiological findings in bouveret's syndrome. Emergency Radiology.2001;8(6):335-337.

9. Grove 0. Acute pyloric obstruction by gallstone: report of a case diagnosed by gastroscopy. Gastrointestinal Endoscopy. 1976;22(4):
212-213.

10. Bedogni G, Contini S, Meinero M, Pedrazzoli C, Piccinini GC. Pyloroduodenal obstruction due to a biliary stone (Bouveret's syndrome) managed by endoscopic extraction. Gastrointestinal Endoscopy.1985; 31(1):36-38.

11. Moschos J, Pilpilidis I, Antonopoulos Z, et al. Complicated endoscopic management of Bouveret's syndrome. A case report and review. Romanian Journal of Gastroenterology. 2005;14(1):75-77.

12. Maiss J, Hochberger J, Muehldorfer S, Keymling J, Hahn EG, Schneider HT. Successful treatment of Bouveret's syndrome by endoscopic laserlithotripsy. Endoscopy. 1999;31(2): S4-S5.

13. Rodríguez-Sanjuán JC, Casado F, Fernández MJ, Morales DJ, Naranjo A. Cholecystectomy and fistula closure versus enterolithotomy alone in gallstone ileus. British Journal of Surgery. 1997;84(5):634-637.

14. Bhama JK, Ogren JW, Lee T, Fisher WE. Bouveret's syndrome. Surgery. 2002;132(1):104-105.

15. Reisner RM, Cohen JR. Gallstone ileus: a review of 1001 reported cases. American Surgeon.1994;60(6):441-446.

16. Zuegel N, Hehl A, Lindemann F, Witte J. Advantages of one-stage repair in case of gallstone ileus. Hepato-Gastroenterology. 1997; 44(13):59-62.

17. Lowe AS, Stephenson S, Kay CL, May J. Duodenal obstruction by gallstone (Bouveret's syndrome): a review of the literature. Endoscopy. 2005;37(1):82-87. 